\title{
THE ORIGIN OF CELESTIAL COORDINATES
}

\author{
C. A. Murray \\ 12 Derwent Road \\ Eastbourne \\ Sussex BN20 7PH \\ England, UK
}

\begin{abstract}
In 1978, Guinot proposed that, for studies of Earth rotation, the zero point of the apparent "right ascension" coordinate on the true equator should be so chosen that the rate of change of its hour angle is exactly proportional to the inertial rate of rotation of the Earth. It has been subsequently suggested that this concept of the "non-rotating origin" supersede the equinox quite generally as the origin of celestial coordinates. Since this proposal was first put forward, there has been much discussion, and some criticism, from Aoki and his colleagues, both published and in private correspondence. Some of the arguments for and against Guinot's proposal are discussed, as a contribution to the wider debate on reference systems now being carried out under the auspices of the IAU.
\end{abstract}

\section{Introduction}

The celestial coordinate system which is currently used for recording observations and in published ephemerides is defined by the instantaneous directions to the celestial pole and the equinox. This coordinate system is, in principle at least, directly accessible to observation with minimum appeal to theory. The direction to the pole is obtained from successive meridian transits of stars at upper and lower culmination, and the direction to the equinox, relative to a priori assumed right ascensions of clock stars, is derived from observations of the Sun or planets relative to these stars.

In practice however, it is not quite as simple as this. Since this instantaneous coordinate system is continually in motion relative to inertial space, it is necessary to have a theory of this motion so that observations even over short periods of time can be referred to a common coordinate system. This requires theories of the Earth's orientation in space and of motions of the Sun and planets.

As the accuracy of observations has increased so has it become necessary to sharpen the geometrical definitions of the system. For example, Atkinson (1973) pointed out that even such a conceptually simple procedure as determining the direction to the instantaneous axis of rotation by observations at upper and lower culmination of the same stars is complicated by short period displacement of that axis within the Earth, and that this can be avoided if instead of the rotation axis one uses a pole which is based on the axis of figure. The pole is implicitly defined by the adopted nutation series; that which has been in use since 1984 (Seidelmann 1982) is based on Atkinson's proposal and is known as the Celestial Ephemeris Pole (CEP).

Another example is the origin of the instantaneous right ascension coordinate. Apparent sidereal time is obtained by comparing observed times of meridian transit with tabulated apparent right

51

J. H. Lieske and V. K. Abalakin (eds.), Inertial Coordinate System on the Sky, 51-59.

(c) 1990 IAU. Printed in the Netherlands. 
ascensions measured from the equinox, which has a secular and oscillatory motion on the true equator due to precession and nutation in right ascension. This was of no practical consequence as long as clocks were not sufficiently precise to detect the shorter period terms. Atkinson and Sadler (1951) pointed out, however, that all the periodic terms would be avoided if, instead of apparent right ascension, the times of meridian transit were measured in terms of mean sidereal time; this is equivalent to adopting as the origin of "right ascension" a point on the equator which is displaced from the true equinox by exactly the amount of the nutation in right ascension.

Even following the proposal of Atkinson and Sadler, mean sidereal time, though sensibly uniform, measures the angular spin of the Earth relative to a moving origin on the equator. In 1978 Guinot (1979) proposed a logical extension of the definition of mean sidereal time to exclude from the origin not only the nutation but also the precession in right ascension. He thus defined the so-called "nonrotating origin" (NRO); the modified sidereal time, or "stellar angle", measured from this origin is a true measure of the Earth's spin.

Since Guinot first proposed the use of the NRO, there has been much discussion and some critcism of it, notably by Aoki and Kinoshita (1982). Although the latest published exposition of the proposal, by Capitaine, Guinot and Souchay (1986), appeared some years ago, the unpublished correspondence has continued at least until the time of the Baltimore IAU meeting in 1988. In this paper I shall, for brevity, refer to these two published papers as AK and CGS respectively, but will not give specific references to the unpublished correspondence.

The whole subject of astronomical reference systems is now under active examination by working groups of the IAU; it therefore seems appropriate that this particular topic should be widely discussed among the astrometric community before any final recommendation is made. The aim of the present paper, therefore, is to try to summarize the main points of the controversy as they now stand, rather than to make a judgement on the proposal. In view of the very extensive correspondence, much of it containing arguments of great mathematical complexity, I hope I may be forgiven if I inadvertently omit points whose significance I may not have fully appreciated.

\section{Theory of the Non-Rotating Origin}

In accordance with the notation used elsewherc (see Murray 1983, Appendix A), the prime ( ${ }^{\prime}$ ) employed here denotes matrix transposition, so that if $\mathbf{a}$ and $\mathbf{b}$ are vectors then $\mathbf{a}^{\prime} \mathbf{b}$ is the same as the dot product $\mathbf{a} \cdot \mathbf{b}$. Since the scalar froduct of two vectors is essentially a transpose matrix multiplication, the prime replaces the more usual "dot" notation. Similarly, the scalar triple product $\mathbf{a} \cdot(\mathbf{b} \times \mathbf{c})=(\mathbf{a} \times \mathbf{b}) \cdot \mathbf{c}$ is written here as $\mathbf{a} \times \mathbf{b}^{\prime} \mathbf{c}$.

Let $\Sigma=\left[\begin{array}{ll}\sigma \quad n \times \sigma & n\end{array}\right]$ be an orthogonal triad of unit vectors, where $\mathbf{n}$ is the direction to the celestial pole, and let $\Omega_{\Sigma}$ be the instantaneous angular velocity of $\Sigma$. If $\dot{n}$ denotes the inertial rate of change of the direction $\mathbf{n}$, we can write

$$
\dot{\mathbf{n}}=\Omega_{\Sigma} \times \mathbf{n} .
$$

If $\frac{\partial}{\partial \tau}$ denotes rate of change relative to the triad $\Sigma$ we have, quite generally, for any vector $\mathbf{g}$,

$$
\dot{\mathbf{g}}=\Omega_{\Sigma} \times \mathbf{g}+\frac{\partial \mathbf{g}}{\partial \tau}
$$

In particular, if $\mathbf{g}$ is perpendicular to $\mathbf{n}$, i.e. it lies in the equator, the component of $\dot{g}$ in the equatorial plane is 


$$
\mathbf{n} \times \mathbf{g}^{\prime} \dot{\mathbf{g}}=\mathbf{n}^{\prime} \Omega_{\Sigma}+\mathbf{n} \times \mathbf{g}^{\prime} \frac{\partial \mathbf{g}}{\partial \tau} .
$$

But since $\dot{n}$ is perpendicular to $n$, the component of $\Omega_{\Sigma}$ parallel to $n$ can be chosen arbitrarily. Guinot (1979) proposed that this should be zero, or

$$
\mathbf{n}^{\prime} \Omega_{\Sigma}=0
$$

in this case, we see from (3), that the angular velocity component about $\mathbf{n}$ relative to the moving frame $\Sigma$, of an equatorial vector, is equal to the same component of its inertial angular velocity. Multiplying (1) vectorially by $n$, and using (4), we see that

$$
\Omega_{\Sigma}=\mathbf{n} \times \dot{\mathbf{n}} .
$$

If $\mathbf{g}$ is now identified with a direction which is fixed in the Earth, such as for example the equatorial point on the prime meridian, the angular velocity of $\mathbf{g}$ relative to the triad $\Sigma$ is the same as the component about $\mathbf{n}$ of the inertial angular velocity of the Earth. The first axis, $\sigma$, of the triad $\Sigma$ is directed toward the NRO.

CGS show that the direction to $\sigma$ can be derived from a knowledge of the instantaneous direction to the pole $\mathbf{n}$ and its trajectory. Let $\Sigma_{\mathrm{o}}$ be a fixed equatorial triad, at some initial epoch, and $\mathbf{n}_{\mathrm{o}}$ be the direction to the corresponding pole. The direction cosines of $\mathbf{n}$ relative to $\Sigma_{\mathrm{o}}$ can be expressed in the form

$$
\mathbf{n}^{\prime} \Sigma_{\mathrm{o}}=\left[\begin{array}{lll}
\sin d \cos E & \sin d \sin E & \cos d
\end{array}\right]
$$

The direction to the node of the instantaneous equator on the equator of $\mathbf{n}_{\mathrm{o}}$ is given by

$$
\mathbf{m}_{1}=\left|\mathbf{n}_{\mathbf{0}} \times \mathbf{n}\right|^{-1} \mathbf{n}_{\mathbf{0}} \times \mathbf{n},
$$

and its longitude along the equator, measured from the first axis of $\Sigma_{\mathrm{o}}$, is $90^{\circ}+E$. From (7) we have

$$
\dot{\mathrm{m}}_{1}=\left|\mathbf{n}_{\mathbf{0}} \times \mathbf{n}\right|^{-1}\left\{\mathbf{m}_{1} \times\left(\mathbf{n}_{\mathbf{0}} \times \dot{\mathbf{n}}\right)\right\} \times \mathbf{m}_{1}=\left|\mathbf{n}_{\mathbf{0}} \times \mathbf{n}\right|^{-1} \mathbf{m}_{1}^{\prime} \dot{\mathbf{n}} \mathbf{n}_{\mathbf{0}} \times \mathbf{m}_{1}
$$

and the inertial rate of change of $\mathbf{m}_{1}$ along the fixed equator of $\mathbf{n}_{\mathbf{0}}$, is then given by

$$
\dot{E}=\mathbf{n}_{0} \times \mathbf{m}_{1}^{\prime} \dot{\mathrm{m}}_{1}=\mid \mathbf{n}_{\mathrm{o}} \times \mathbf{n}^{-1} \mathbf{m}_{1}^{\prime} \dot{\mathbf{n}} \text {. }
$$

The rate of change of $\mathbf{m}_{1}$ relative to the triad $\Sigma$ is given by

$$
\frac{\partial}{\partial \tau} \mathbf{m}_{1}=\dot{m}_{1}-\Omega_{\Sigma} \times \mathbf{m}_{1},
$$

and hence the angular rate of change of $\mathbf{m}_{1}$ along the instantaneous equator, relative to the $\mathrm{NRO}$, can be written

$$
\dot{E}+\dot{s}=\mathbf{n} \times \mathbf{m}_{1} \cdot \frac{\partial}{\partial \tau} \mathbf{m}_{1}
$$

where 


$$
\dot{s}=-\left|1+n^{\prime} n_{0}\right|^{-1} n_{0} \times n^{\prime} \dot{n} .
$$

Thus, at any time $\tau$, the longitude of $\mathbf{m}_{1}$ measured along the instantaneous equator from the NRO is given by

$$
90^{\circ}+E+s=90^{\circ}+E+\int_{0}^{\tau} \dot{s} \mathrm{~d} \tau .
$$

The location of the NRO can therefore be determined in principle only from a knowledge of the direction of the pole $\mathbf{n}$ and its past history to time $\tau$.

The hour angle, $\theta$, of the NRO is the stellar angle and its time derivative is the inertial angular velocity component of the Earth about $\mathbf{n}$. It can be expressed in the form

$$
\theta=A+\lambda
$$

where $A$ is the instantaneous ascension of a star on the meridian through longitude $\lambda$.

\section{Relationship between the Non-Rotating Origin and the Equinox}

Let $\mathbf{N}=[\mathbf{l} \mathbf{~ m ~} \mathbf{n}]$ be the instantaneous equatorial triad in which $\mathbf{l}$ is directed toward the true equinox and $\mathbf{m}=\mathbf{n} \times \mathbf{l}$. If $\Omega_{\mathrm{N}}$ denotes the instantaneous inertial angular velocity of $\mathbf{N}$, due to precession and nutation, we have

$$
\dot{\mathrm{n}}=\Omega_{\mathrm{N}} \times \mathbf{n},
$$

hence, from (5),

$$
\Omega_{\Sigma}=\Omega_{\mathrm{N}}-\mathbf{n} \mathbf{n}^{\prime} \Omega_{\mathrm{N}}
$$

The inertial rate of change of the direction of the true equinox is

$$
\mathrm{i}=\Omega_{\mathrm{N}} \times \mathbf{l}
$$

and its component in the equator is

$$
m^{\prime} \mathbf{i}=n^{\prime} \Omega_{N},
$$

which represents the instantaneous rate of change of the equinox direction along the equator due to the combined effects of precession and nutation in right ascension.

But since $l$ is an equatorial vector, its angular velocity relative to the moving frame $\Sigma$ is equal to its inertial angular velocity component about $\mathbf{n}$. Hence we also have

$$
\mathbf{m}^{\prime} \frac{\partial \mathbf{l}}{\partial \tau}=\mathbf{n}^{\prime} \Omega_{\mathrm{N}} \text {. }
$$

The right ascension of $\sigma$ is then 


$$
\alpha(\sigma)=-\int_{0}^{\tau} n^{\prime} \Omega_{\Omega} d \tau
$$

and is the total displacement of the equinox due to the accumulated precession in right ascension from epoch $\tau=0$, and the equation of the equinoxes.

The Greenwich Sidereal Time is

$$
\varphi=\alpha+\lambda
$$

where $\alpha$ is the right ascension of a star on the meridian through longitude $\lambda$ and is related to the stellar angle by

$$
\theta=\varphi-\alpha(\sigma)
$$

Therefore, from (14), instantaneous ascension is related to right ascension by

$$
A=\alpha-\alpha(\sigma) .
$$

\section{Evaluation of $\alpha(\sigma)$}

We can express the angular velocity vector $\Omega_{\mathrm{N}}$ in the form

$$
\Omega_{\mathrm{N}}=\dot{\chi} \mathbf{n}_{\mathrm{m}}-\dot{\psi} \mathbf{k}-\Delta \dot{\psi} \mathbf{k}+\dot{\varepsilon}_{\mathrm{m}} \mathbf{l}_{\mathrm{m}}-\dot{\varepsilon} \mathbf{l}
$$

where $\dot{\chi}$ is the rate of planetary precession on the mean equator, $\dot{\psi}$ is the rate of precession in longitude on the ecliptic of date, (luni-solar minus geodesic precession), $\Delta \dot{\psi}$ denotes the rate of change of nutation in longitude, $\dot{\varepsilon}_{\mathrm{m}}, \dot{\varepsilon}$ are the rates of change of mean and true obliquity respectively, and the unit vectors $k, l_{m}$ and $n_{m}$ are directed toward the pole of the ecliptic, the mean equinox and celestial pole of date.

If $\mathbf{K}_{\mathrm{m}}=\left[\begin{array}{lll}\mathbf{l}_{\mathrm{m}} & \mathbf{j}_{\mathrm{m}} & \mathbf{k}\end{array}\right]$ is the ecliptic triad of date we have

$$
\mathbf{n}_{\mathrm{m}}=\sin \varepsilon_{\mathrm{m}} \mathbf{j}_{\mathrm{m}}+\cos \varepsilon_{\mathrm{m}} \mathbf{k}
$$

and

$$
\mathbf{n}=\sin \varepsilon \sin \Delta \psi \mathbf{l}_{\mathrm{m}}+\sin \varepsilon \cos \Delta \psi \mathbf{j}_{\mathrm{m}}+\cos \varepsilon \mathbf{k},
$$

and hence

$$
n^{\prime} \Omega_{\mathrm{N}}=-(\dot{\psi}+\Delta \dot{\psi}) \cos \varepsilon+\dot{\chi}\left(\sin \varepsilon_{\mathrm{m}} \sin \varepsilon \cos \Delta \psi+\cos \varepsilon_{\mathrm{m}} \cos \varepsilon\right)+\dot{\varepsilon}_{\mathrm{m}} \sin \varepsilon \sin \Delta \psi .(27)
$$

This equation is quite rigorous. It is easy to show that it is equivalent to the equation (A2-32) of $\mathrm{AK}$, when expanded to the second order in $\Delta \psi$ and $\Delta \varepsilon$, where 


$$
\Delta \varepsilon=\varepsilon-\varepsilon_{\mathrm{m}}
$$

Expanding (27) to the first order in $\Delta \psi, \Delta \varepsilon$, and neglecting $\dot{\chi}$ and $\dot{\varepsilon}_{\mathrm{m}}$ which arise from the motion of the ecliptic, we obtain the approximation

$$
n^{\prime} \Omega_{\mathrm{N}} \approx-\dot{\psi} \cos \varepsilon_{\mathrm{m}}-\frac{\mathrm{d}}{\mathrm{d} \tau}\left(\Delta \psi \cos \varepsilon_{\mathrm{m}}\right)+(\dot{\psi}+\Delta \dot{\psi}) \Delta \varepsilon \sin \varepsilon_{\mathrm{m}} .
$$

AK express the right ascension of $\sigma$ in the form

$$
\alpha(\sigma)=q+(\Delta q)_{s}+(\Delta q)_{p}
$$

where, to the approximation in (29),

and

$$
\begin{aligned}
& q=\int_{0}^{\tau} \cos \varepsilon_{\mathrm{m}} \mathrm{d} \psi, \\
& (\Delta q)_{\mathrm{p}}=\Delta \psi \cos \varepsilon_{\mathrm{m}}-\left[\int_{0}^{\tau}(\dot{\psi}+\Delta \dot{\psi}) \Delta \varepsilon \sin \varepsilon_{\mathrm{m}} d \tau\right]_{\mathrm{p}}
\end{aligned}
$$

$$
(\Delta q)_{s}=-\left[\int_{0}^{\tau} \Delta \dot{\psi} \Delta \varepsilon \sin \varepsilon_{m} d \tau\right]_{s} ;
$$

the subscripts $\mathrm{p}$ and $\mathrm{s}$ denoting periodic and secular terms respectively.

Equation (31) represents accumulated precession in right ascension. (It should be noted that in our notation $\psi$ is the luni-solar precession on the ecliptic of date.)

Equation (32) is a modified equation of the equinoxes; the first term represents the first order expression, although the Astronomical Ephemeris gives $\Delta \psi \cos \varepsilon$.

AK make the point that the second order terms in (32) and (33) introduce both secular and periodic terms which have not hitherto been included in the calculation of the precession in RA and the equation of the equinoxes, although Woolard (1953) gave approximate values. Retaining only the largest terms it is easily shown that

$$
(\Delta \mathrm{q})_{\mathrm{p}}=\Delta \psi \cos \varepsilon_{\mathrm{m}}+0.00264 \sin \Omega+0.000063 \sin 2 \Omega
$$

where $\Omega$ is the longitude of the Moon's node, and

$$
(\Delta q)_{s}=-0.00385 \tau
$$

where $\tau$ is measured in centuries. AK gave -0.00388 for this coefficient, but Aoki later revised it to -0:00386; I find the value given here, which is derived from six periodic terms in the nutation series (Seidelmann 1982), and agrees with that given by CGS.

\section{Discussion}

The coordinate triad $\Sigma$ has apparent theoretical advantages over the traditional celestial coordinate 
system, $\mathbf{N}$, in that it depends formally on the direction of a single vector $\mathbf{n}$ and its instantaneous rate of change $\dot{n}$. It does not explicitly involve either the ecliptic or the equinox. We have seen in Section 2 , above, how the direction to $\sigma$ can be derived only from a knowledge of the direction of the pole relative to a fixed coordinate system based on the pole $\mathbf{n}_{\mathrm{o}}$ at at some initial epoch. The direction to the pole at any instant relative to the fixed system must still be obtained from the theory of precession and nutation. Among the objections which have been raised against the proposal for the NRO by AK is that the motion of the pole is governed entirely by the action of the Moon and Sun on the Earth, and hence depends implicitly on the ecliptic as well as the equator; and therefore the supposed independence of $\sigma$ from the location of the ecliptic is more apparent than real. In any case, the direction cosines of the pole $\mathbf{n}$ relative to the fixed frame $\Sigma_{\mathrm{o}}$ must be calculated from the usual precession and nutation quantities.

A practical difficulty with the current system is that the position of the ecliptic, and hence that of the equinox, is not well determined relative to the stars. However Aoki has pointed out that, in the foreseeable future, observations of pulsar positions by timing, combined with conventional interferometric positions, are likely to improve considerably the location of the ecliptic relative to the equatorial coordinate system.

We now discuss some of the specific points which have been raised in the controversy.

\subsection{Definttion of THE CELESTIAL POLE}

One of the criticisms raised by $\mathrm{AK}$, and repeated in subsequent unpublished correspondence, concerns the choice of pole for the reference system $\Sigma$. In the discussion following his original paper, Guinot said "I believe now that Atkinson's pole is the best"; in other words he supported the use of the CEP rather than the pole of rotation as the pole of the triad $\Sigma$. However CGS state "The pole of instantaneous rotation is the one which logically agrees with the following theoretical developments" and later "In principle, we should use here the coordinates of the pole of instantaneous rotation". It is clear from the development above in Section 2 that the choice of pole is irrelevant; at no point in the argument do we make any appeal to the angular velocity vector of the Earth. AK make the point, originally made by Atkinson that, by using the CEP, the "dynamical variation of latitude" is eliminated and furthermore that the spin about the CEP is theoretically constant, as I have also stressed elsewhere (Murray 1983), whereas the resultant total angular velocity of the Earth is not; it is thus exactly correct to use the CEP and not the rotation pole. This has now been accepted by Guinot and Capitaine.

\subsection{MOTION OF THE NRO IN SPACE}

Aoki makes the point that the name "non-rotating origin" gives a false impression because the NRO has a secular and oscillatory motion in space; he prefers the name "departure point", which is in accordance with the usual meaning of that term in celestial mechanics. It is easy to show that, while the right ascension of a fixed object increases by $2 \pi$ during a complete precession period, the instantaneous ascension only increases by $2 \pi \cos \varepsilon$. But there is also a more subtle point concerning the small secular term $(\Delta q)_{s}$ which comes from second order nutation. By considering the average over all right ascensions of fixed objects close to the equator, Aoki shows that the instantaneous ascension increases on the average by

$$
\frac{1}{2} \int_{0}^{\tau}(\Delta \varepsilon \Delta \dot{\psi}-\Delta \psi \Delta \dot{\varepsilon}) \sin \varepsilon_{n} d \tau
$$


which is exactly equal to $-(\Delta q)_{s}$; therefore this cancels in right ascension but not in instantaneous ascension.

This demonstrates that the reference frame based on the concept of the NRO is purely local, and has a net rotation relative to inertial space. This is accepted by Guinot and Capitaine, but it does not invalidate the principle that the stellar angle measured from the NRO is proportional to the orientation of the Earth about the CEP.

\subsection{Computational PRoblems}

Among several computational difficulties, Aoki draws attention to the fact that mixed secular terms, namely periodic terms with secularly increasing coefficients, appear in $s$, and also in the nutation relative to the NRO, and that these are undesirable. Also, the node of the instantaneous equator on the fixed equator, $\mathbf{m}_{1}$, which is used as an intermediate reference point in deriving $\sigma$, is numerically unstable because of the small inclination, $d$, between the two equators. Guinot and Capitaine however reject these points as giving no real computational problems.

\subsection{Change of Precession}

One of the ideas behind Guinot's proposal is that the instantaneous ascension of an equatorial object will be unaffected by a change in the value of the precession, but Aoki points out that, away from the equator, an error in precession will introduce spurious proper motions in both coordinates.

\section{The Space Reference System}

Guinot's original paper contained recommendations for the definition and realization of the space reference system. The essential points may be summarized as follows:

The basic system should be defined by a catalogue of positions (and proper motions) of suitable objects; ideally these should be extra-galactic objects such as quasars whose proper motions can be assumed to be negligible.

The directions of the poles of the equator and ecliptic should be given explicitly as functions of time in this system. If subsequent observations show that the assumed coordinates of either pole relative to the basic system require correction, then the system should not be changed but corrections applied to the numerical expressions for the pole directions.

The NRO was apparently only considered by Guinot in connection with the instantaneous equatorial reference system and not specifically as a substitute for the equinox in any fixed epoch equatorial system. This is however envisaged by CGS who state that it "could in a general manner replace advantageously the equinox especially for observations which are not sensitive to the orientation of the ecliptic" (my italics).

In considering the possible implications for astronomy of redefining the right ascension coordinate, one should bear in mind what has already happened in the analogous case of the mean sidereal time proposal of Atkinson and Sadler. Nearly forty years later, published ephemerides still give apparent right ascensions referred to the true equinox! However, ephemeris computations for special purposes such as time services use mean sidereal time. It is after all merely a matter of computational 
convenience.

Guinot's suggestion that the directions to the poles be given explicitly in terms of the time is an excellent one which I also have advocated (Murray 1983). Nutation relative to the moving mean equatorial triad is already given in the form of harmonic series, but precession is concealed in elaborate trigonometric formulae and associated angular variables which disguise very effectively what is after all a simple physical phenomenon. This has been admirably demonstrated by Fabri (1980) who obtained numerical expressions for the direction cosines of the poles of the mean equator and of the ecliptic, by direct integration of the equations of motion of the poles in vector form. An example of the inadequacy of the traditional trigonometric representation is that the rate of luni-solar precession in longitude is given along the fixed ecliptic whereas the precessional motion of the mean celestial pole $\mathbf{n}$ is physically an instantaneous rotation about the pole of the ecliptic of date, $\mathbf{k}$. This is not to imply that computations based on the traditional formulation are incorrect, but rather that they conceal unnecessarily the true physical situation.

In discussing revision of the astronomical reference system it is important to distinguish between fundamental changes, such as the proposal to define it by positions of extra-galactic objects rather than the kinematics of the Earth, and changes of procedure which are for computational convenience in particular applications, such as the form of presentation of the coordinate transformation for precession and nutation, or indeed the adoption of the NRO. Nevertheless, anything which makes fundamental astrometry more transparent to non-specialists is to be welcomed.

\section{References}

Aoki, S., and Kinoshita, H.: 1982, Celes. Mech., 29, 335.

Atkinson, R.d'E.: 1973, Astron. J., 78, 147.

Atkinson, R.d'E., and Sadler, D H.: 1951, Mon. Not. Roy. Astron.Soc. 111, 619.

Capitaine, N., Guinot, B., and Souchay, J.: 1986, Celes. Mech., 39, 283.

Fabri, E.: 1980, Astron. Astrophys., 82, 123.

Guinot, B.: 1979, Time and the Earth's Rotation, 7, D.D. McCarthy, J.D. Pilkington (eds.)

D Reidel Publishing Co., Dordrecht.

Murray, C.A.: 1983, Vectorial Astrometry, Adam Hilger, Bristol.

Seidelmann, P.K.: 1982, Celes Mech. 27, 79.

Woolard, E.W.: 1953, Astron. Pap. Amer. Ephem. 15, Part 1, 165.

\section{Discussion}

KOPEJKIN: How do you define the notion of inertial space? I know that general relativity gives some ambiguities in this notion.

MuRRAY: I realize that in a relativistic context inertial space is difficult to define, but in this paper I have only been concerned with a purely classical problem.

STANDISH: The locations and motions of the equatorial plane and the ecliptic are now determined strictly from ranging observations of the Moon coupled with the lunar dynamics. The orientation of these planes in the ephemerides no longer depends upon the optical observations.

MURRAY: The problem is how to relate optical and radio observations of objects other than the Moon or planets to these well-defined planes. 